\title{
SOME REMARKS ON THE LOCATION OF FIXED POINTS
}

\author{
MICHAEL EDELSTEIN and DARYL TINGLEY
}

(Received 14 June 1983)

Communicated by R. Vyborny

\begin{abstract}
Several procedures for locating fixed points of nonexpansive selfmaps of a weakly compact convex subset of a Banach space are presented. Some of the results involve the notion of an asymptotic center or a Chebyshev center.

1980 Mathematics subject classification (Amer. Math. Soc.): 47 H 09, 47 H 10.

Throughout this paper $X$ denotes a uniformly convex Banach space, $K$ a convex set and $f: K \rightarrow K$ a nonexpansive mapping. In [3] it is shown that the asymptotic center (see below) of each bounded sequence of iterates is a fixed point under $f$. In this paper we consider the fixed point properies associated with closely related centers; those that arise when sequences of subsets, rather than singletons, are considered.
\end{abstract}

Let $\left\{S_{n}\right\}$ be a sequence of sets in a Banach space $Y$ with $\cup S_{n}$ bounded and let $K$ be a nonempty closed convex subset of $Y$. For each $n=1,2, \ldots$ and each $x \in K$ set

$$
r_{n}(x)=\sup \left\{\|x-y\|: y \in \bigcup_{m \geqslant n} S_{m}\right\}
$$

This research was supported by NSERC Grant A-3999.

(c) 1984 Australian Mathematical Society 0263-6115/84\$A2.00+0.00 


$$
\begin{gathered}
r_{n}=\inf \left\{r_{n}(y): y \in K\right\}, \\
r(x)=\inf \left\{r_{n}(x): n=1,2, \ldots\right\}, \\
r=\inf \{r(y): y \in K\} .
\end{gathered}
$$

The set $c_{n}=\left\{x \in K: r_{n}(x)=r_{n}\right\}$ is frequently referred to as the Chebyshev center (of $\mathrm{U}_{m \geqslant n} S_{m}$ ) with respect to $K$. A familiar fact about Chebyshev centers is that they are nonempty convex sets whenever $Y$ is reflexive or $K$ is weakly compact. In certain Banach spaces (see [2]), uniformly convex ones included, the Chebyshev centers are known to be singleton sets.

More recently (see [8]) the set $c=\{x \in K: r(x)=r\}$ has been introduced and termed the asymptotic center with respect to $K$, whilst the number $r$ is called the asymptotic radius (of the sequence of sets or singletons with respect to $K$ ). Clearly

$$
r(c)<r(x)
$$

whenever $x \in K \backslash\{c\}$.

To simplify notation we shall write $r\left(\left\{S_{n}\right\}, k\right)$ and $\mathscr{A}\left(\left\{S_{n}\right\}, K\right)$ for the asymptotic radius and center respectively of $\left\{\mathrm{U}_{m \geqslant n} S_{m}\right\}$ with respect to $K$.

Arguments such as those used in [3] and [4] show that in a uniformly convex Banach space the Chebyshev centers $\left\{c_{n}\right\}$ converge to the asymptotic center $\mathscr{A}\left(\left\{S_{n}\right\}, K\right)$.

\section{3}

3.1 THEOREM 1. Let $S$ be a non-empty subset of a uniformly convex Banach space $X$, and let $f: X \rightarrow X$ be nonexpansive. If $\cup\left\{f^{n}[S]\right\}$ is bounded then $c$, the asymptotic center of $\left\{f^{n}[S]: n=1,2 \ldots\right\}$ with respect to $X$, is a fixed point of $f$, that is $f(c)=c$.

Furthermore, if $\xi$ is any other fixed point of $f$, then

$$
\begin{aligned}
& \inf \left\{\sup \left\{\|x-c\|: x \in f^{n}[S]\right\}: n=1,2, \ldots\right\} \\
& \quad<\inf \left\{\sup \left\{\|x-\xi\|: x \in f^{n}[S]\right\}: n=1,2, \ldots\right\}
\end{aligned}
$$


Proof. For $n \geqslant 2$ we have

$$
\begin{aligned}
r_{n}(f(c)) & =\sup \left\{\|f(c)-y\|: y \in \bigcup_{m \geqslant n} f^{m}[S]\right\} \\
& =\sup \left\{\|f(c)-f(z)\|: z \in \bigcup_{m \geqslant n} f^{m-1}[S]\right\} \\
& \leqslant \sup \left\{\|c-z\|: z \in \bigcup_{m \geqslant n-1} f^{m}[S]\right\} \\
& =r_{n-1}(c) .
\end{aligned}
$$

Thus $r_{n}(f(c)) \leqslant r_{n-1}(c)$ for each $n$, and hence $r(f(c)) \leqslant r(c)$. However as $r(c)=r\left(f^{m}[S], X\right)$ it follows that $f(c)$ is in $\mathscr{A}\left(f^{m}[S], X\right)$ and, by remarks in Section 2, that $f(c)=c$.

To prove (6) first observe that for any fixed point $z$ of $f$,

$$
\begin{aligned}
\sup \left\{\|x-z\|: x \in f^{n}[S]\right\} & =\sup \left\{\|f(x)-f(z)\|: x \in f^{n-1}[S]\right\} \\
& \leqslant \sup \left\{\|x-z\|: x \in f^{n-1}[S]\right\},
\end{aligned}
$$

implying that

$$
\sup \left\{\|x-z\|: x \in \bigcup_{n \geqslant m} f^{n}[S]\right\}=\sup \left\{\|x-z\|: x \in f^{m}[S]\right\} .
$$

Thus (6) is equivalent to $r(c)<r(\xi)$, which is true by (5).

Let $X, S$, and $f$ be as in the above theorem.

3.2 If $\varnothing \neq S \subseteq X$ and $S$ is bounded with $f[S] \subseteq S$, then obviously $\bigcup\left\{f^{n}[S]\right.$ : $n \geqslant m\}=f^{m}[S]$. We conclude that if $c_{m}$ is the Chebyshev center of $f^{m}[S]$ then $c=\lim c_{m}$ exists and is a fixed point of $f$.

3.3 Theorem 1 can be restated in terms of a mapping $f: K \rightarrow K$ where $K$ is a closed and bounded convex set in $X$. In this case the asymptotic center is taken with respect to $K$. One could also relax the nonexpansive condition on $f$ in a manner similar to that employed in [3].

4.1 THEOREM 2. Let $S$ be a nonempty subset of a uniformly convex Banach space $X$, and let $f: X \rightarrow X$ be nonexpansive. If $\bigcup\left\{f^{n}[S]\right\}$ is bounded and for some natural 
$k$ and $l$ we have $r_{k}=r_{k+l}$, then $r_{k}=r_{k+1}=\cdots=r_{k+l}$ and $f\left(c_{k}\right)=c_{k}=\cdots=$ $c_{k+l}$; hence also $f\left(c_{k+n}\right)=c_{k+n}$ for $n=1,2, \ldots, l$. (Here $r_{i}$ and $c_{i}$ are as in Section 2.)

ProOF. Clearly $r_{k} \geqslant r_{k+1} \geqslant \cdots \geqslant r_{k+l}$, so $r_{k}=r_{k+1}=\cdots=r_{k+l}$. It suffices then to prove the theorem for $l=1$. Now $c_{k+1}$ is the unique point with the property that $\bigcup_{n \geqslant k+1} f_{n}[S] \subseteq B\left(c_{k+1}, r_{k+1}\right)$. However

$$
f\left(\bigcup_{n \geqslant k} f^{n}[S]\right)=\bigcup_{n \geqslant k+1} f^{n}[S] \subseteq \bigcup_{n \geqslant k} f^{n}[S] \subseteq B\left(c_{k}, r_{k}\right)=B\left(c_{k}, r_{k+1}\right)
$$

showing that $c_{k}=c_{k+1}$. Since $f$ is nonexpansive,

$$
\sup \left\{\left\|f\left(c_{k}\right)-f(y)\right\|: y \in \bigcup_{n \geqslant k} f^{n}[S]\right\} \leqslant \sup \left\{\left\|c_{k}-y\right\|: y \in \bigcup_{n \geqslant k} f^{n}[S]\right\}=r_{k} .
$$

Thus $\bigcup_{n \geqslant k+1} f^{n}[S] \subseteq B\left(f\left(c_{k}\right), r_{k}\right)=B\left(f\left(c_{k}\right), r_{k+1}\right)$, showing that $f\left(c_{k}\right)=c_{k+1}$ $=c_{k}$.

4.2 Under the hypothesis of Theorem 2, a finite (rather than infinite, as in Theorem 1) procedure leads to the location of a fixed point. As the following example shows the fixed point obtained may, or may not coincide with that obtained via the asymptotic center.

EXAMPLE. Let $K=\left\{(x, y, z) \in R^{3}: z \geqslant 0, x^{2}+y^{2}+z^{2} \leqslant 1\right\}$ and define $f$ : $K \rightarrow K$ by $f((x, y, z))=(y, 0, z)$. Clearly $f$ is nonexpansive. The sets $K$ and $f[K]$ both have Chebyshev radius 1 and Chebyshev center $(0,0,0)$. For $n \geqslant 2 f^{n}[K]$ has Chebyshev radius $1 / 2$ and center $(0,0,1 / 2)$, showing the asymptotic center of $f^{n}[K]$ is $(0,0,1 / 2)$. Thus Theorems 1 and 2 may both be used to locate a fixed point of $f$, however the fixed points obtained do not coincide.

4.3 The assumption that $X$ is uniformly convex is used only to guarantee the existence of a unique asymptotic center for $\left\{f^{n}[S]\right\}$ in Theorem 1 , and of a unique Chebyshev center for each $f^{n}[S], k \leqslant n \leqslant k+l$, in Theorem 2 . Any other condition leading to the existence and uniqueness of such centers may be substituted for uniform convexity; (see [2], [9]).

4.4 Theorem 2 can also be restated in terms of a mapping $f: K \rightarrow K$ where $K$ is a closed bounded convex set in $X$. In this case the Chebyshev centers and radii would be taken with respect to $K$. A result of Floret [6], Theorem 2, shows that if $\overline{c o}(f[K])=K$ (where $\overline{c o}$ denotes the closed convex hull) then the Chebyshev center of $K$ is a fixed point of $f$. If $\overline{c o}(f[K])=K$ the Chebyshev radii of $\overline{c o}(f[K])$ and $K$ are equal, thus in view of the remarks in 4.3, his result follows from a theorem similar to Theorem 2. 
The concept of asymptotic regularity is due to Browder and Petryshyn [1]. In [5] it was modified to uniform asymptotic regularity. If $S$ is a subset of a Banach space $Y$, the mapping $h: S \rightarrow S$ is said to be uniformly asymptotically regular if $\left\|h^{n+1}(x)-h^{n}(x)\right\| \rightarrow 0$ uniformly over $S$. It was shown there that if $K$ is convex and bounded and $g: K \rightarrow K$ is nonexpansive then $f=\frac{1}{2}(g+I)$ is uniformly asymptotically regular (here $I$ is the identity mapping). The mappings $f$ and $g$ have identical fixed point sets. Procedures for locating fixed points are much simpler for uniformly asymptotically regular maps, as shown by the next result.

5.1 THEOREM 3. Let $h: S \rightarrow S$ be a uniformly asymptotically regular self-mapping of a set $S$ in a Banach space $Y$. Then $S_{\omega}=\cap\left\{h^{n}[S]: n=1,2, \ldots\right\}$ is the fixed point set of $h$.

Proof. Let $F$ be the fixed point set of $h$. Certainly $F \subseteq S_{\omega}$. To show that $S_{\omega} \subseteq F$ let $\xi \in S_{\omega}$ and suppose, for a contradiction, that $h(\xi) \neq \xi$. Let $\varepsilon=\| h(\xi)$ $-\xi \|$ and let $n$ be sufficiently large to imply that $\left\|h^{n+1}(x)-h^{n}(x)\right\|<\varepsilon$ for all $x \in S$. Let $x \in S$ be such that $h^{n}(x)=\xi$. Then $\varepsilon=\|h(\xi)-\xi\| \leqslant\left\|h^{n+1}(x)-h^{n}\right\|$ $<\varepsilon$, a contradiction.

5.2 Corollary 1. Let $K$ be a closed bounded convex set in a uniformly convex Banach space $X$, let $f: K \rightarrow K$ be nonexpansive and uniformly asymptotically regular, and let $\varnothing \neq S \subseteq K_{\omega}=\cap\left\{f^{n}[K]: n=1,2, \ldots\right\}$. Then the Chebyshev center of $S$ with respect to $K$ is a fixed point of $f$, and hence is in $K_{\omega}$.

Proof. As $f[S]=S$ this follows from Theorem 2.

5.3 COROLlaRY 2. Let $K$ be a weakly compact convex subset of a Banach space $Y$, and $f: K \rightarrow K$ be nonexpansive. Suppose in addition that $K$ has normal structure (see [7]). By a theorem of Kirk [7] the set of fixed points $F$ of $f$ is non-empty. Then $g=\frac{1}{2}(f+I)$ is uniformly asymptotically regular, and applying Theorem 3 to $g, K_{\omega}=F$ is the fixed point set of both $f$ and $g$.

5.4 AN EXAMPLE. It is tempting to assume that in Theorem 3 the sets $f^{n}[K]$, in some sense, converge to $K_{\omega}$ and in particular that the Chebyshev radii of $f^{n}[K]$ converge to the Chebyshev radius of $K_{\omega}$. The following example shows this is not the case. 
Let $K$ be the unit ball in the Hilbert space $l^{2}$. For $x=\left(x_{k}\right)=$ $\left\langle x_{1}, x_{2}, \ldots, x_{k}, \ldots\right\rangle \in K$ set

$$
f(x)=\left\langle\left(1-\frac{1}{k}\right) x_{k}\right\rangle .
$$

Clearly $f$ is nonexpansive, $f(K) \subseteq K$, and $f^{n}(x)=\left\langle\left(1-\frac{1}{k}\right)^{n} x_{k}\right\rangle$. Also

$$
\left\|f^{n+1}(x)-f^{n}(x)\right\|^{2}=\sum\left(1-\frac{1}{k}\right)^{2 n}\left(\left(1-\frac{1}{k}\right) x_{k}-x_{k}\right)^{2} \leqslant \sum\left(1-\frac{1}{k}\right)^{2 n}\left(\frac{1}{k}\right)^{2} .
$$

It is readily seen that the above tends to 0 as $n \rightarrow \infty$.

Let $e_{k}=\langle\underset{k-1 \text { times }}{0,0, \ldots, 0,1,0}\rangle$. Then

$$
\left\|f^{n}\left(e_{k}\right)\right\|=\left(1-\frac{1}{k}\right)^{n} \text {. }
$$

Thus letting $k^{\prime}$ be such that for all $k \geqslant k^{\prime},\left(1-\frac{1}{k}\right)^{n} \geqslant 1-\varepsilon$, it follows that $\left\|f^{n}\left(e_{k}\right)\right\| \geqslant 1-\varepsilon$ for $k \geqslant k^{\prime}$. It can now be concluded that the Chebyshev radius of $f^{n}(K)$ is 1 for all $n$. However $\cap f^{n}[K]=\{0\}$.

Note that this example can easily be modified so that $\cap f^{n}[K]$ is more than a singleton.

6.1 In this section we present a procedure for locating a fixed point $o$ a nonexpansive map $f$ from a weakly compact convex set $K$ with normal structure to itself, without passing to an alternative map (for example, $\frac{1}{2}(I+f)$ ). In general this method involves a transfinite process.

Let $K_{0}=K$ and suppose that for each ordinal $\beta<\alpha, K_{\beta}$ has been defined such that $f\left(K_{\beta}\right) \subseteq K_{\beta}$ and $K_{\beta}$ is a weakly compact convex subset of $K_{\gamma}$, for all $\gamma<\beta$.

$$
K_{\alpha}= \begin{cases}\mathscr{A}\left(\left\{f^{n}\left(K_{\alpha-1}\right)\right\}, K_{\alpha-1}\right), & \alpha \text { not a limit ordinal, } \\ \bigcap_{\beta<\alpha} K_{\beta}, & \alpha \text { a limit ordinal }\end{cases}
$$

It is shown in [8] that if $x \in K$ then the asymptotic center of $\left\{f^{n}(x)\right\}$ is mapped by $f$ into itself. With essentially the same proof it can be shown that the same is true for an arbitrary set $\varnothing \neq S \subseteq K$. That is

$$
f\left(\mathscr{A}\left(\left\{f^{n}[S]\right\}, K\right)\right) \subseteq \mathscr{A}\left(\left\{f^{n}[S]\right\}, K\right)
$$

We conclude (in either case) that $f\left(K_{\alpha}\right) \subseteq K_{\alpha}$. Also, by properties of the asymptotic center stated in Section 2 and properties of the intersection, in either case $K_{\alpha}$ is a weakly compact convex subset of $K_{\beta}$, for all $\beta \subset \alpha$. 
It is also shown in [8] that if $C$ is convex with normal structure, contains more than one point, and $C \supseteq B_{n} \supseteq B_{n+1}, n=1,2, \ldots$, then $\mathscr{A}\left(\left\{B_{n}\right\}, C\right)$ is a proper subset of $C$. It follows that for each ordinal $\alpha, K_{\alpha}$ is a proper subset of $K_{\beta}, \beta<\alpha$ unless $K_{\beta}$ is a singleton and hence there is a smallest ordinal $\lambda$ such that $K_{\lambda}$ is a singleton. We conclude with

THEOREM 4. With $f$, and $\lambda$ as above, the singleton $K_{\lambda}$ is a fixed point of $f$.

6.2 Theorem 4 can be restated in a form similar to that of Theorems 1 and 2. Let $K \subseteq Y$ be such that either $K$ is closed and $Y$ is reflexive or $K$ is weakly compact. Let $f: K \rightarrow K$ and assume that $S \subseteq K$ is such that $\cup f^{n}[S]$ is bounded. In either case, the asymptotic center of $\left\{f^{n}[S]\right\}, K_{0}$, is a weakly compact subset of $Y$ with $f\left(K_{0}\right) \subseteq K_{0}$. Hence the above procedure can be applied to this choice of $K_{0}$, rather than $K$ itself.

6.3 As in Theorem 1, the fixed point obtained by the above procedure may be characterized as follows.

THEOREM 5. Let $f, K, K_{\alpha}$ and $K_{\lambda}$ be as above and let $K_{\lambda}=\{c\}$. Then for every fixed point $\xi$ of $f$, and each ordinal $\alpha$,

$$
\begin{aligned}
& \inf \left\{\sup \left\{\|x-c\|: x \in f^{n}\left[K_{\alpha}\right]\right\}: n=1,2, \ldots\right\} \\
& \quad \leqslant \inf \left\{\sup \left\{\|x-\xi\|: x \in f^{n}\left[K_{\alpha}\right]\right\}: n=1,2, \ldots\right\} .
\end{aligned}
$$

Proof. Let $\alpha$ be fixed. Then the left side of (7) is $r(c)$ and the right side is $r(\xi)$, where $r(x)$ is defined by (3) with $B_{n}=f^{n}\left[K_{\alpha}\right]$. As $c \in K_{\alpha+1}=\mathscr{A}\left(f^{n}\left[K_{\alpha}\right], K_{\alpha}\right)$, $r(c)=r$ and the theorem follows from (4).

\section{References}

[1] F. E. Browder and W. V. Petryshyn, "The solution by iteration of nonlinear functional equations in Banach spaces', Bull. Amer. Math. Soc. 72 (1966), 571-575.

[2] M. M. Day, R. C. James and S. Swaminathan, 'Normed linear spaces that are uniformly convex in every direction', Canad. J. Math 23 (1971), 1051-1059.

[3] M. Edelstein, 'The construction of an asymptotic center with a fixed point property', Bull. Amer. Math. Soc. 78 (1972), 206-208.

[4] M. Edelstein, 'Fixed point theorems in uniformly convex Banach spaces', Proc. Amer. Math. Soc. 44 (1974), 369-374.

[5] M. Edelstein and R. C. O'Brien, 'Nonexpansive mappings, asymptotic regularity, and successive approximations', J. London Math. Soc. (2) 17 (1978), 547-554. 
[6] Klaus Floret, 'Eine Bemerkung über a-priori-Fixpunkte nichtexpansiver Abbildungen', Manuscripta Math. 6 (1972), 321-326.

[7] W. A. Kirk, 'A fixed point theorem for mappings which do not increase distances', Amer. Math. Monthly 72 (1965), 1004-1006.

[8] T. C. Lim, 'Characterizations of normal structure', Proc. Amer. Math. Soc. 43 (1974), 313-319.

[9] J. Reinermann and R. Shoenberg, 'Some results in the fixed point theory of nonexpnsive mappings and generalized contractions', Proc. Sem. Fixed Point Theory and its Applications, Dalhousie University, Halifax, N. S., Canada, 1975 (Academic Press).

Department of Mathematics, Statistics and Computing Science

Dalhousie University

Halifax, N. S. B3H 4H8

Canada 